\title{
CONTROLE QUÍMICO DE DOENÇAS FOLIARES EM MILHO
}

\author{
NICÉSIO FILADELFO JANSSEN DE ALMEIDA PINTO ${ }^{1}$ \\ ${ }^{1}$ Pesquisador, Embrapa Milho e Sorgo. Caixa Postal 151, CEP. 35701-970 Sete Lagoas, MG. E- mail: \\ nicesio@cnpms.embrapa.br (autor para correspondência).
}

Revista Brasileira de Milho e Sorgo, v.3, n.1, p.134-138, 2004

RESUMO - O objetivo deste trabalho foi avaliar a eficiência de fungicidas no controle das doenças foliares do milho provocadas por Phaeosphaeria maydis, Exserohilum turcicum e Puccinia polysora. Os seguintes fungicidas $\left(\mathrm{g}\right.$ i.a. $\left.\mathrm{ha}^{-1}\right)$ foram aplicados nas cultivares BR 3123, BR 3101 e IAEN-I, com pulverizador costal, com início no estádio de polinização: tebuconazole (200), mancozeb (2400), tebuconazole + mancozeb $(200+2400)$, sulfato de estreptomicina + oxitetraciclina $(450+45)$, propiconazole $(250)$, azoxystrobin (150), imibenconazole (150), triforine (285), prochloraz (450), carbendazim (350), chlorothalonil (1125). Parcelas sem fungicida constituíram a testemunha. O modelo experimental foi de blocos ao acaso, com 36 tratamentos em três repetições, sendo os fungicidas aleatorizados entre as parcelas e as cultivares dentro das parcelas. Foram realizadas três pulverizações, com intervalos de dez dias. A avaliação sintomatológica foi realizada no estádio de grão pastoso, usando-se escala de notas de 0 a 5 ( $0=$ ausência de lesões ou pústulas foliares e $5=$ lesões em $100 \%$ das folhas, com seca das plantas). Verificou-se que, para o controle da mancha de Phaeosphaeria maydis, foram eficientes os fungicidas mancozeb e azoxystrobin; para a queima de Exserohilum turcicum, foram eficientes o tebuconazole, o imibenconazole, o sulfato de estreptomicina + oxitetraciclina, o triforine e o prochloraz; para a ferrugem de Puccinia polysora, o fungicida azoxystrobin foi altamente eficiente, seguido por tebuconazole, tebuconazole + mancozeb e imibenconazole.

Palavras-chave: Zea mays, Exserohilum turcicum, Phaeosphaeria maydis, Puccinia polysora, fungicidas.

\section{CHEMICAL CONTROL OF FOLIAR DISEASES IN CORN}

ABSTRACT - This work was carried out to evaluate the efficiency of several fungicides in the
control of foliar diseases incited by Phaeosphaeria maydis, Exserohilum turcicum and
Puccinia polysora in corn fields. The following fungicides $(\mathrm{g}$ a.i. ha-1 $)$ were applied in the
cultivars BR 3123, BR 3101 and IAEN-I, with a back sprayer, beginning in the pollination
stage: tebuconazole (200), mancozeb $(2400)$, tebuconazole + mancozeb $(200+2400)$,
streptomycin sulphate + oxytetracycline $(450+45)$, propiconazole $(250)$, azoxystrobin $(150)$,
imibenconazole (150), triforine $(285)$, prochloraz (450), carbendazim (350), chlorothalonil
(1125). Plots without fungicide constituted the control. The experimental model used was
randomized blocks with 36 treatments in 3 replications, being the fungicidal randomized among
the plots and the cultivars inside the plots. Three sprayings were carried out at 10-day intervals.
The symptomatological evaluation was accomplished in the pasty grain stage, being given scores
from 0 to 5 ( 0 = absence of lesions or pustules foliate and $5=$ lesions in $100 \%$ of the leaves,
with drought of the plants). It was verified that for the control of the spot of Phaeosphaeria
maydis were most efficient the fungicides mancozeb and azoxystrobin; for the control of the 
blight of Exserohilum turcicum were most efficient the fungicides tebuconazole, imibenconazole, streptomycin sulphate + oxytetracycline, triforine and prochloraz; and for the control of the rust of Puccinia polysora the fungicide azoxystrobin was highly efficient, proceeded by tebuconazole, tebuconazole + mancozeb and imibenconazole.

Key words: Zea mays, Exserohilum turcicum, Phaeosphaeria maydis, Puccinaia polysora, fungicides.

A partir da década de 90, algumas doenças fúngicas foliares, pelo aumento da freqüência e da severidade com que vêm ocorrendo, têm causado sensível redução qualitativa e quantitativa na produção de milho. Essas doenças são: a mancha foliar provocada por Phaeosphaeria maydis, as ferrugens provocadas por Puccinia sorghi, Puccinia polysora e Phyzopella zeae e a queima de turcicum (Exserohilum turcicum).

Vários fatores podem estar contribuindo para o aumento na incidência de doenças na cultura do milho: o aumento da área cultivada; o aumento do número de cultivares comerciais com diferentes níveis de resistência às doenças; o manejo inadequado de água em plantios sob pivô ou na aspersão convencional, os plantios diretos de milho sobre restos culturais de milho e os plantios consecutivos de milho durante o ano todo, os quais podem contribuir para aumentos significativos de patógenos (Pinto et al., 1997).

A queima da folha do milho causada por Exserohilum turcicum (Helminthosporium turcicum, Setosphaeria turcica) constitui um dos problemas fitossanitários que afetam a cultura do milho. Segundo Pinto (1980), as cultivares de milho pipoca são altamente suscetíveis a esse patógeno. Quando os sintomas da doença aparecem antes do pendoamento, os prejuízos podem ser elevados (Issa, 1983). Segundo Pereira et al. (1993), a doença causada pelo fungo Exserohilum turcicum reduziu a produção e aumentou o tombamento das plantas, quando comparado com o controle nos híbridos testados. De acordo com Issa (1983), a aplicação dos fungicidas maneb $+\mathrm{Zn}$, captafol e chlorotalonil, em plantas de milho pipoca da cultivar South American Mushroom, foi eficiente no controle de E. turcicum, sendo que o controle da doença incrementou a produção em até $66,0 \%$. Ademais, Nowell \& Laing (1998) relatam que, em cultivares de milho doce, a queima foliar de E. turcicum pode ser controlada por diversos fungicidas, especialmente os sistêmicos dos grupos químicos dos triazóis (tebuconazole e propiconazole), em combinações com benzimidazóis (carbendazim) e, quando usados separadamente, os fungicidas benzimidazóis e triazóis também foram efetivos. Esses autores também observaram que os fungicidas de ação por contato, como mancozeb e chlorotalonil, promoveram controle significativo, quando comparados com a testemunha não pulverizada.

Galli et al. (1993) aplicaram Folicur 250 PM (tebuconazole), nas doses de 0,75 e 1,0 kg do produto comercial/ha, via pulverização foliar, para o controle da ferrugem polissora do milho, causada por Puccinia polysora, e observaram que esse fungicida reduziu significativamente o número de pústulas por folha.

Segundo Borges \& Campos (1995), o fungicida fentin hydroxyde mostrou-se o mais eficiente no controle da mancha de Phaeosphaeria maydis, cujas plantas não apresentaram sintomas; sendo seguido pelo mancozeb, propiconazole e tebuconazole, cujas plantas apresentaram lesões esparsas. Pinto \& Fernandes (1995) avaliaram o controle de Phaeosphaeria maydis com os fungicidas triadimenol, tiofanato metílico, chlorotalonil, benomyl, tebuconazole, oxicloreto de cobre, trifenil hidróxido de estanho (fentin hydroxyde) e iprodione. 
O fungicida oxicloreto de cobre mostrou-se o mais eficiente no controle desse patógeno, seguido pelo trifenil hidróxido de estanho. Entretanto, estes dois fungicidas apresentaram fitotoxicidade às plantas de milho. De acordo com Pinto (1999), o controle efetuado por mancozeb sobre a mancha foliar do milho provocada por Phaeosphaeria maydis resultou em aumento de $63,1 \%$ na produção de grãos em relação à testemunha sem fungicida. Além disso, segundo Pinto (1997), o fungicida tebuconazole foi eficiente no controle da queima de Exserohilum turcicum e da ferrugem comum do milho (Puccinia sorghi), enquanto que o mancozeb foi eficiente no controle da mancha foliar por Phaeosphaeria maydis.

Este trabalho teve como objetivo avaliar o controle químico das doenças foliares em milho provocadas por Phaeosphaeria maydis, Exserohilum turcicum e Puccinia polysora, devido ao surgimento de novos produtos ainda não testados em trabalhos anteriores.

O experimento foi conduzido em 1999, em um latossolo vermelho-escuro álico, textura argilosa, fase cerrado, de relevo suave ondulado, cultivado por vários anos com milho, na Embrapa Milho e Sorgo, em Sete Lagoas, MG, tendo como coordenadas geográficas $19^{\circ} 28^{\prime} 00^{\prime \prime}$ de latitude sul e $44^{\circ} 15^{\prime} 08^{\prime}$ 'W. Grw., a uma altitude média de $732 \mathrm{~m}$. O clima da região, segundo classificação de Koeppen, é do tipo Aw, ou seja, clima de savana de inverno seco. A temperatura média anual é de 22,1 ${ }^{\circ} \mathrm{C}$, com amplitude de variação anual em torno de 5 ${ }^{\circ} \mathrm{C}$. Registra uma precipitação média anual elevada $(1.340 \mathrm{~mm})$, sendo o período chuvoso em dezembro (305 mm) e o seco em agosto $(6,3 \mathrm{~mm})$.

Para a realização deste trabalho, utilizaramse as cultivares de milho BR 3123, BRS 3101 e IAEN-I, semeadas em parcelas constituídas por seis fileiras de sete metros de comprimento, com espaçamento de 1,0 metro entre as fileiras e 0,2 m entre plantas. Os seguintes fungicidas (g i.a. ha-1) foram avaliados: tebuconazole (200), mancozeb (2400), tebuconazole + mancozeb $(200+2400)$, sulfato de estreptomicina + oxitetraciclina $(450+45)$, propiconazole (250), azoxystrobin (150), imibenconazole (150), triforine (285), prochloraz (450), carbendazim (350), chlorothalonil (1125). Parcelas sem fungicida constituíram a testemunha. Esses fungicidas foram aplicados com pulverizador costal manual, com início no estádio de polinização das cultivares, cujas plantas apresentavam sintomas iniciais das doenças em estudo, as quais ocorreram por infecção natural.

O modelo experimental foi o de blocos ao acaso, com 36 tratamentos em três repetições, sendo os fungicidas aleatorizados entre as parcelas e as cultivares dentro das subparcelas (12 parcelas fungicidas e três subparcelas - cultivares). Foram realizadas três pulverizações, com intervalos de dez dias. A avaliação sintomatológica foi realizada no estádio de grão pastoso, usando-se escala de notas de 0 a 5, proposta por Pinto \& Fernandes (1995) $\{0=$ ausência de lesões; $1=$ lesões esparsas; $2=1$ esões em $50 \%$ das folhas e com $25 \%$ de severidade; $3=$ lesões em $75 \%$ das folhas e com $50 \%$ de severidade; $4=$ lesões em $100 \%$ das folhas e com $75 \%$ de severidade; e $5=$ lesões em $100 \%$ das folhas e com seca total das plantas\}.

No período de execução do experimento, a umidade relativa média do ar foi de $69,3 \%$, enquanto que as médias das temperaturas máxima, mínima e média foram de 29,$2 ; 17,3$ e $22,3{ }^{\circ} \mathrm{C}$, respectivamente. Nesse período, além da suplementação de água na cultura, ocorreu uma precipitação pluviométrica de 518,2 $\mathrm{mm}$.

É oportuno ressaltar que nenhum dos tratamentos fungicidas apresentou fitotoxicidade às plantas de milho e que as três cultivares diferiram entre si quanto às suas reações aos patógenos avaliados. A cultivar BR 3123 foi a mais suscetível à mancha de 
Phaeosphaeria maydis, a cultivar BRS 3101 a mais suscetível à ferrugem polissora e a cultivar IAEN-I a mais suscetível à queima por turcicum. Devido à baixa severidade de cada uma dessas cultivares às demais doenças, as reações apresentadas na Tabela 1 referem-se apenas às cultivares mais suscetíveis em cada patossistema estudado.

Os resultados da avaliação da eficiência dos fungicidas no controle das doenças causadas por Phaeosphaeria maydis, Exserohilum turcicum e Puccinia polysora estão contidos na Tabela 1, onde verifica-se que, para o controle da mancha de Phaeosphaeria maydis, foi eficiente o fungicida mancozeb, o que está em conformidade com os resultados obtidos por Pinto $(1997,1999)$ e também o azoxystrobin. Com relação ao fungicida tebuconazole, este não apresentou efeito sobre a doença, sendo o resultado semelhante ao apresentado pela testemunha, o que está em conformidade com aqueles obtidos por Pinto (1997) e Pinto \& Fernandes (1995), mas que não coadunam com os resultados apresentados por Borges \& Campos (1995). Para o controle da queima de Exserohilum turcicum, foram mais eficientes tebuconazole, imibenconazole, sulfato de estreptomicina + oxitetraciclina, triforine e prochloraz. A eficiência de tebuconazole no controle dessa doença foi relatada por Issa (1983), Pinto (1997) e também corrobora os relatos de Nowell \& Laing (1998), os quais afirmam que os fungicidas do grupo químico dos triazóis, ao qual pertence o tebuconazole, foram eficientes no controle desse patógeno. Para o controle da ferrugem de Puccinia polysora, o fungicida azoxystrobin foi altamente eficiente, seguido por tebuconazole, tebuconazole + mancozeb e imibenconazole. A eficiência de tebuconazole no controle dessa ferrugem também foi relatado por Galli et al. (1993).

Do exposto, pode-se concluir que: 1 - Os fungicidas mancozeb e azoxystrobin são eficientes

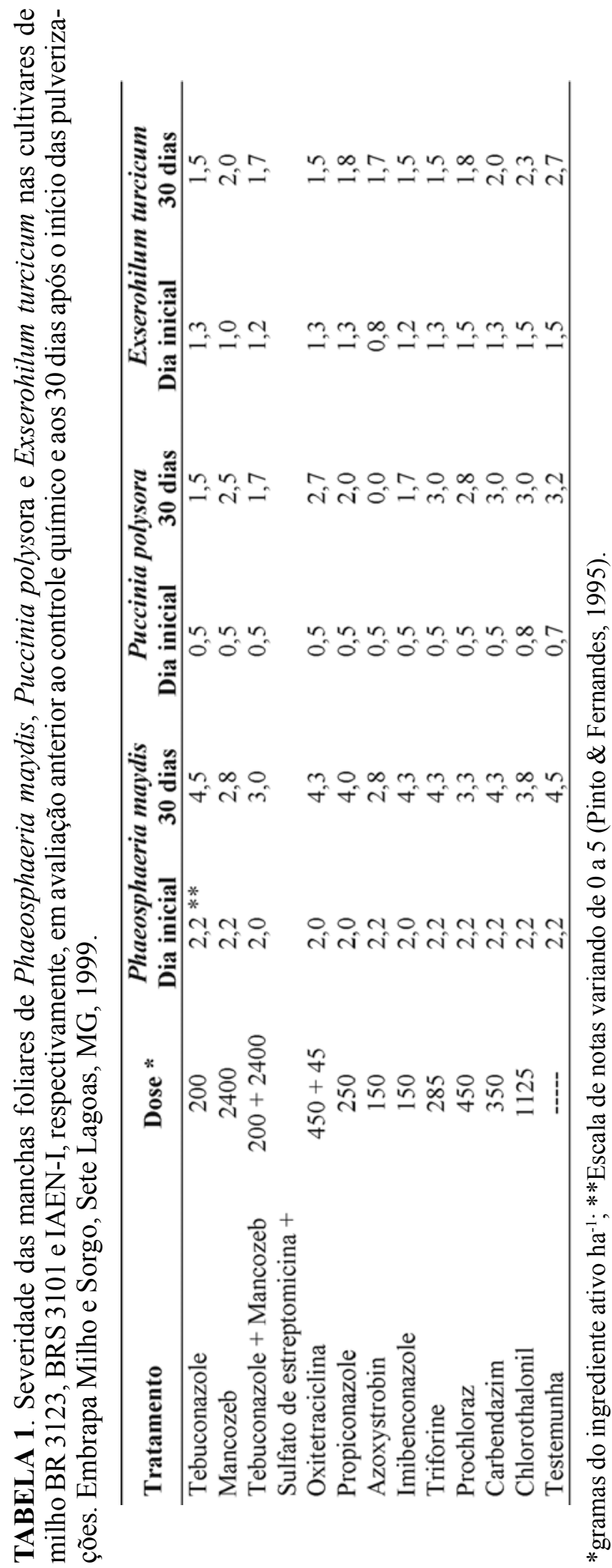


no controle da mancha de Phaeosphaeria maydis; 2 - Os fungicidas tebuconazole, imibenconazole, sulfato de estreptomicina + oxitetraciclina, triforine e prochloraz são eficientes no controle da queima de Exserohilum turcicum; e 3 - Os fungicidas azoxystrobin, tebuconazole, tebuconazole + mancozeb e imibenconazole são eficientes no controle da ferrugem causada por Puccinia polysora.

\section{Agradecimentos}

A Osni Alves da Silva, José Moreira Campos e Ademar Verneque (Assistentes de Pesquisa), a Gilberto Ribeiro Rodrigues e Almir Roberto da Silva (Operários Rurais) a valiosa contribuição na condução dos experimentos que resultaram neste trabalho.

\section{Literatura Citada}

BORGES, A. L.; CAMPOS, H. D. Avaliação de fungicidas no controle de mancha na folha do milho causado por Phaeosphaeria maydis. Fitopatologia Brasileira, Brasília, v. 18, p. 333, 1995. Suplemento.

GALli, M. A.; SALVO, S.; CERVO, J. C.; PARADELLA, A. Avaliação da eficiência de fungicidas sistêmicos no controle da ferrugem (Puccinia polysora Underw.) na cultura do milho (Zea mays L.). Fitopatologia Brasileira, Brasília, v18, p310, 1993. Suplemento.

ISSA, E. Controle químico de Helminthosporium turcicum Pass. em milho pipoca, Zea mays L. $\mathbf{O}$ Biológico, Campinas, v. 49, n. 2, p. 41 - 43, 1983.

NOWELL, D. C.; LAING, M. D. Evaluation of fungicides to control Exserohilum turcicum on sweet corn in South Africa. Journal of the Southern Africa Society for Horticultural Sciences, Stellenbosch, v. 8, n. 2, p. 65 - 69, 1998.

PEREIRA, O. A. P.; SILVA, H. P.; REZENDE, I. C. Perda da produção de milho causada por Exserohilum turcicum (Pass.) Leonard \& Sugss. Fitopatologia Brasileira, Brasília, v. 18, p. 333, 1993. Suplemento.

PINTO, N. F. J. A. Doenças do Milho. Informe Agropecuário, Belo Horizonte, v. 6, n. 72, p. 50 53, 1980 .

PINTO, N. F. J. A. Eficiência de fungicidas no controle de doenças foliares do milho. Summa Phytopathologica, Jaboticabal, v. 23, n. 3/4, p. 271 - 274, 1997.

PINTO, N. F. J. A. Eficiência de doses e intervalos de aplicações de fungicidas no controle da manchafoliar do milho provocada por Phaeosphaeria maydis Rane, Payak \& Renfro. Ciências e Agrotecnologia, Lavras, v. 23, n. 4, p. 1006 1009, 1999.

PINTO, N. F. J. A.; FERNANDES, F. T.; OLIVEIRA, E. Milho (Zea mays L.): Controle de doenças. In: VALE, F. X. R.; ZAMBOLIM, L. (Ed.). Controle de doenças de plantas: Grandes Culturas. Viçosa: UFV, 1997. cap. 17, p. 821 - 863.

PINTO, N. F. J. A.; FERNANDES, F. T. Avaliação de fungicidas no controle da mancha foliar do milho causada por Phyllosticta sp. (Phaeosphaeria maydis). Fitopatologia Brasileira, Brasília, v. 20, p. 333, 1995. Suplemento. 